\title{
Probing Protein Structures in Solution by Molecular Dynamics Simulation and Small-Angle X-ray Scattering
}

\author{
Hsiao-Ching Yang ${ }^{1}$, Shang-Wei Lin ${ }^{1}$, Yung-Chi Ge ${ }^{1}$, Ming-Yi Huang ${ }^{1}$, Cheng-Han Yang ${ }^{1}$, Wei-Min Liu ${ }^{1}$, \\ Anthony P. Duff ${ }^{2}$, Chun-Ming Wu ${ }^{3}$, Yi-Kang Lan ${ }^{4}$, An-Chung Sư ${ }^{4}$, Yi-Qi Yeh ${ }^{3}$, U-Ser Jeng ${ }^{3,4}$, Pi-Tai Chou $^{5}$ \\ ${ }^{1}$ Department of Chemistry, Fu Jen Catholic University, Xinzhuang 24205, Taiwan, \\ ${ }^{2}$ National Deuteration Facility, Australian Nuclear Science and Technology Organisation, Lucas Heights, NSW 2234, Australia, \\ ${ }^{3}$ National Synchrotron Radiation Research Center, Hsinchu Science Park, Hsinchu 30076, Taiwan, \\ ${ }^{4}$ Department of Chemical Engineering, National Tsing Hua University, Hsinchu 30013, Taiwan, \\ ${ }^{5}$ Department of Chemistry, National Taiwan University, Taipei City 10617, Taiwan.
}

hcyang_chem@mail.fju.edu.tw

Lore of chemical biology guides us that drug discovery of protein binding relies on either optimize the active site complexity of lock and key or induced-fit with conformation selection dynamics; yet, the latter that often-coupled protein interior transport dynamics was much harder to study due to its lack of strong interactions in transient states.[1-2] This study starts to make progress in using in-situ operando X-ray and neutron contrast variation techniques to depict the landscape of protein binding substrate dynamics in solution. We herein demonstrate, for the first time, the 3-D dynamical structures of hydrated CYP450 protein exterior surfaces to interior buried heme site by a distributed connection of channels that direct the reactant in and out. Using CYP450s of prostacyclin synthase (PGIS) and thromboxane synthase (TXAS) as prototypes we have unveiled the unique dynamics of P450 functional channels in/out the haem site, which drive a variety of water molecules motion, water density change and pre-organization toward the heme active site and hence harness the substrate binding selectivity. The result is able to clarify how these two proteins catalyze the same substrate of prostaglandin $\mathrm{H}_{2}$ by entirely different regio-chemical-selective pathways.

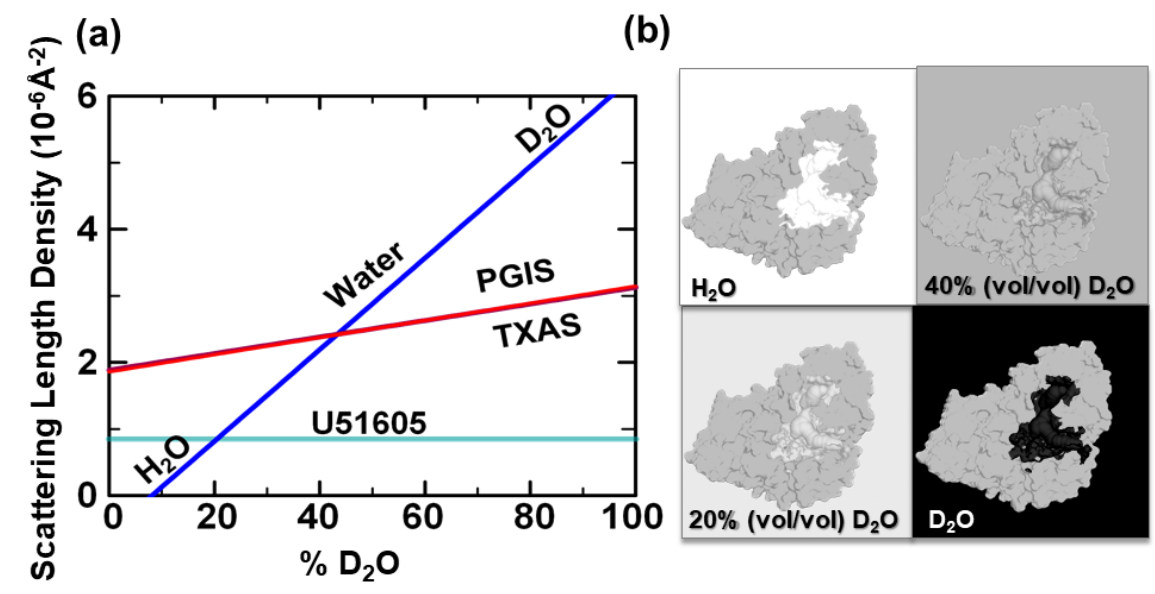

Figure 1. (a) The SLD value of our protein PGIS, TXAS and the small molecule. (b) Principle of contrast matching in non-deuterated protein. Illustrated here is a protein/channel complex (gray surface and gradient colored surface, respectively)

[1] Yang, H.-C., Ge, Y.-C., Yang, C.-H. \& Chao, W.-C. (2018). ACS Catal. 8, 2534.

[2] Yang, H.-C., Yang, C.-H., Huang, M.-Y., Lu, J.-F., Wang, J.-S., Yeh, Y.-Q. \& Jeng, U. S. (2018). J. Phys. Chem. B, $121,11229$.

Keywords: Cytochrome P450; substrate channel; molecular dynamics simulation; small-to-medium-angle X-ray scattering; and neutron contrast variation scattering.

This work was supported by grants from the Ministry of Science and Technology of Taiwan (Grant Nos. MOST 109-2113-M-030-002). 